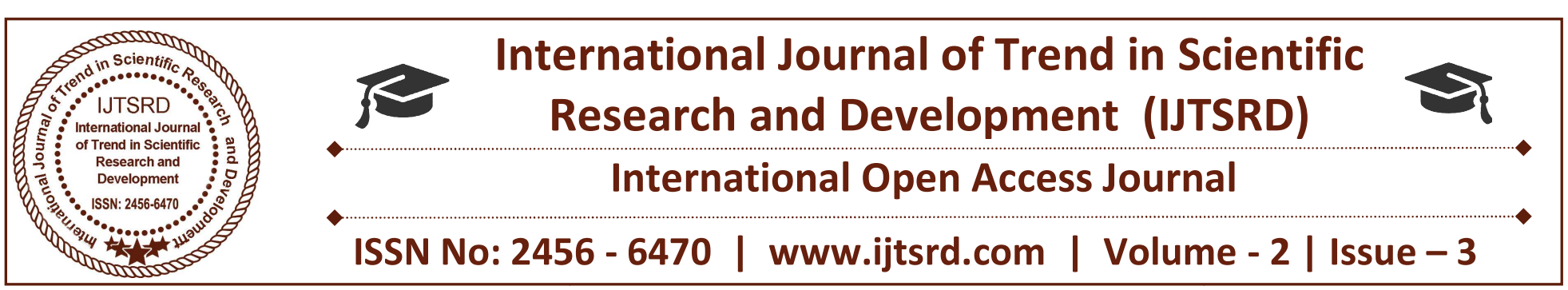

\title{
Collaborative Filtering with Preventing Fake Ratings
}

\author{
${ }^{1}$ Dr. A. Srinivasa Rao, B.Bhagyalakshmi, Ab. Sirajunnisa, Md. Ashraf, E. Harika, Ch. Gangadhar \\ ${ }^{1}$ Professor \\ Department of Computer Science \& Engineering, \\ Dhanekula Institute of Engineering and Technology
}

Vijayawada, Andhra Pradesh, India

\begin{abstract}
Social voting is a promising new feature in online social networks. It has distinctive challenges and opportunities for suggestion. In this paper, we increase a set of matrix factorization (MF) and nearest-neighbor (NN)-based recommended systems (RSs) that explore user social network and group association information for social voting recommendation. During experiments with actual social voting traces, we express that social network and group association information can drastically progress the popularity-based voting advice, and social network in order dominates group association sequence in NN-based approaches. We as well observe that social and group information is much more precious to cold users than to heavy users. In our experiments, simple meta path based nearestneighbor models outperform computationconcentrated on matrix factorization models in hotvoting recommendation, while user's preferences for non-hot votings can be better mined by matrix factorization models. We further put forward a hybrid $\mathrm{RS}$, bagging distinct single approaches to get the best top-k hit rate.
\end{abstract}

KEYWORDS: Social-voting, Recommendations, Matrix-factorization, Nearest-neighbor, collaboration, E-Commerce

\section{INTRODUCTION}

Online social networks, such as Facebook and Twitter, facilitate easy information sharing among associates. A consumer not only can share his/her updates, in forms of text, image, and video, with his/her direct friends, but also, can hastily disseminate those updates to a much larger viewer of indirect friends, leveraging on the rich connectivity and global reach of popular online social networks. Many online social networks now offer the social voting function, through which a user can share with friends his/her opinions, e.g., like/dislike, on different subjects, ranging from customer statuses, display pictures, to games played, products purchased, websites visit, etc. Taking like/dislike type of votings one step further, some online social networks, e.g., Sina Weibo, empower users to initiate their own voting campaigns, on any topic of their interests, with user customized voting options. The friends of a voting initiator can participate in the campaign or retweet the campaign to their friends. Other than stimulating social interactions, social voting also has many potential commercial values. Advertisers can initiate votings to advertise certain brands. Product managers can initiate votings to conduct market research. Ecommerce owners can strategically launch votings to attract more online customers.

Social voting conducts unique challenges and opportunities for Recommender organizations utilizing social conviction information. Furthermore, voting participation data are binary without negative samples. It is, therefore, intriguing to develop Recommender organizations for social voting. Toward addressing these challenges, we progress a set of novel Recommender organizations models, together with matrix-factorization -based models and nearest-neighbor -based models, to learn user-voting 
comforts by concurrently mining evidence on uservoting sharing, user to user friendship, and user group association. We methodically estimate and associate the presentation of the anticipated models using actual social voting suggestions composed from SinaWeibo. The influence of this paper is threefold.

1) Online social voting has not been much reviewed to user/admin information. We progress matrixfactorization -based and nearest-neighbor-based Recommender organizations models. We show through experiments with real social voting suggestions that both social network evidence and group association information can be extracted to significantly progress the accuracy of approval-based voting recommendation.

2) Our research on nearest-neighbor-based models instruct that social network suggestion group reminder social and group information is more valued to cold users than to hefty users. 1

3) We show that simple meta-path based nearestneighbor models outstrip calculation-intensive matrixfactorization models in hot-voting recommendation, while users' safeties for non-hot voting's can be better mined by matrix-factorization models.

\section{RELATED WORK}

Bond et al. [1] conducted a 61-million-person about social influence on Facebook [24] during the 2010 congressional elections in U.S. They established that strong ties in research online social networks can influence people's approval of voting actions. Different from [1], we study social influence on user's adoption of online social voting's, which are originated and broadcast purely in online social networks. Collaborative filtering-based Recommender organizations use user response data to forecast user benefits, leading to very exact recommendations [2][11].Adomavicius and Tuzhilin [2] presented a survey of Recommender organizations. Korean [4], [5] and Salakhutdinov and Mnih [7] proposed MF-based models for rating prediction. Cremonesi et al. [10] and Shi et al[13]. studied collaborative filtering for top-k recommendation. Rendle et al[12]. presented a generic optimization criterion Bayesian Personalized Ranking-Optimization derived from the maximum posterior estimator for optimal personalized ranking. Rendle et al[12]. proposed a generic learning algorithm Learn BPR to optimize Bayesian Personalized Ranking-Optimization. Bayesian Personalized Ranking can work on top of our proposed methods, such as Weibo-matrixfactorization and nearest-neighbor approaches to optimize their performance. The increasingly popular online social networks provide additional information to enhance pure rating-based RSs. There are many previous studies concerning how to integrate social network information to increase recommendation accuracy, just to name a few, Ma et al.[14] proposed to factorize user-item rating matrix and user to user relationship matrix together for item rating prediction. Metal claimed that a user's ratings of an item is influenced by his/her friends. A user's rating to an item consists of two parts, the user's own rating of the item and the user's friends' ratings of the item. The authors then proposed to combine the two ratings linearly to get a final forecast rating. Jamali and Ester[15] claimed that a user's interest is influenced by his/her friends. Thus, a user's latent feature is constrained to be like his/her friends' buried features in the process of matrix-factorization. Yang et al[16]. claimed that a user's interest is multi fact and planned to split the limited social network into circles. Different circles are used to predict ratings of items in different categories. Jiang et al[17]. addressed exploiting information from multiple platforms to recognize user's needs in a comprehensive way. They proposed a semi managed transfer learning method in Recommender administration address the problem of cross-platform conduct forecast, which fully activities the small number of overlapped crowds to bridge the information across different stands. Jiang et al[17]. considered enriching evidence for accurate user-item link prediction by representing a social network as a star-structured hybrid graph centered on a social domain, which connects with other item domains to help improve the prediction accuracy. One-class collaborative filtering deals with binary rating data, reflecting a user's action or not. In One-class collaborative filtering, only positive samples are observed, and there are many missing entries.

One-class collaborative filtering deals with secondary rating data, reflecting a user action or not. In one-class collaborative filtering, only positive samples are experimental, and there is large quantity of hidden entries. one-class collaborative filtering has been widely deliberate. In this paper can also be classified as one-class collaborative filtering. The differences that we are commerce with secondary data from numerous channels, involving secondary user-voting events, user to user trust relationships, and user to group association. 


\section{PROPOSED WORK}

We consider top-k voting recommendation in online social networks. For each user, the recommender system has suggested a small number, say $k$, of votings from all existing votings. Matrix factorization approaches were found to be very competent in general top- $k$ recommendation. Moreover, social network evidence can be demoralized to improve the accuracy of top- $k$ recommendation. For this reason, we start with matrix factorization approaches using both social network evidence and group association information. We propose a multi-channel matrix factorization model, which factorizes user-voting relations, use to user and user to group interactions concurrently, gearing to improve top- $k$ hit rate. We first develop neighborhoods by navigating different types of metapaths in the Weibo heterogeneous information network. We then explore user neighborhoods in the hidden feature space derived from matrix factorization models.

\section{Nearest-Neighbor Method}

Other than matrix factorization approaches, nearestneighbor -based recommendations have also been considered. nearest-neighbor methods are generally used in recommended system. Thus, it is very captivating to study the presentation of nearestneighbor copies on social voting recommendation delinquent. In nearest-neighbor -based methods, the neighborhood of a operator can be calculated using collaborative filtering, or it can be a set of directly or indirectly associated friends in a social network, or just a set of users with analogous interests in a similar group. This makes it suitable to combine social trust and user-group relationship into nearest-neighbor founded top- $k$ recommendation.

\section{Screenshot}

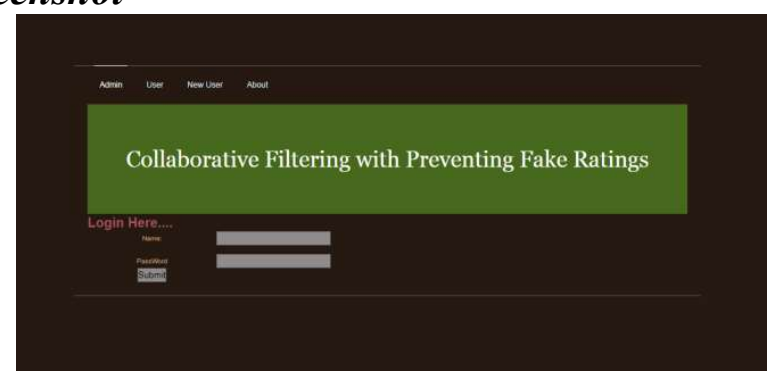

\section{CONCLUSION AND FUTURE WORK}

In this paper, we present a set of matrix factorizationbased and nearest neighbor-based and recommendation organization for online social voting. During researches with actual data, we found that social network and group association information can significantly improve the precision of approval-based voting recommendation, especially for cold users, and social network information directly to group affiliation information in nearest neighbor-based approaches. In this paper demonstrated that social and group information is much more valuable to progress recommendation precision for cold users than for heavy users. This is since cold users tend to contribute in popular voting's. In our experiments, simple Meta path-based nearest neighbor models outperform computation intensive Matrix factorization models in hot-voting commendation, while users' interests for non-hot voting's can be better mined by Matrix factorization models. This paper is only for step toward thorough study of social voting recommendation. As an instantaneous imminent work item, we would like to refer how voting contented information can be mined for recommendation, especially for cold voting's. We are also attentive in developing voting recommendation organization customized for individual users, given the convenience of multi-channel evidence about their social neighborhood activities.

\section{REFERENCES}

1) R. M. Bond et al., "A 61-million-person experiment in social influence and political mobilization," Nature, vol. 489, pp. 295-298, Sep. 2012.

2) G. Adomavicius and A. Tuzhilin, "Toward the next generation of recommender systems: A survey of the state-of-the-art and possible extensions," IEEE Trans. Knowl. Data Eng., vol. 17, no. 6,pp. 734-749, Jun. 2005.

3) X. Su and T. M. Khoshgoftaar, "A survey of collaborative filtering techniques," Adv. Artif. Intell., vol. 2009, Aug. 2009, Art. no. 421425, doi: $10.1155 / 2009 / 421425$.

4) Y. Koren, "Factorization meets the neighborhood: A multifaceted collaborative filtering model," in Proc. ACM KDD, 2008, pp. 426-434. 
5) Y. Koren, "Collaborative filtering with temporal dynamics," in Proc.KDD, Paris, France, 2009, pp. 447-456.

6) A. Paterek, "Improving regularized singular value decomposition for collaborative filtering," in Proc. KDDCup, 2007, pp. 39-42.

7) Salakhutdinov and A. Mnih, "Probabilistic matrix factorization," in Proc. NIPS, vol. 20. 2008, pp. 1257-1264.

8) K. Yu, A. Schwaighofer, V. Tresp, X. Xu, and H.P.Kriegel, "Probabilistic memory-based collaborative filtering," IEEE Trans. Knowl. Data Eng., vol. 16, no. 1, pp. 56-69, Jan. 2004.

9) R. H. Keshavan, A. Montanari, and S. Oh, "Matrix completion from noisy entries," J. Mach. Learn. Res., vol. 11, pp. 2057-2078, Jul. 2010.

10) P. Cremonesi, Y. Koren, and R. Turrin, "Performance of recommender algorithms on top$\mathrm{N}$ recommendation tasks," in Proc. ACM RecSys, 2010, pp. 39-46.

11) Y. Zhang, B. Cao, and D.-Y. Yeung, "Multidomain collaborative filtering," in Proc. 26th Conf. Uncertainty Artif. Intell. (UAI), Catalina Island,CA, USA, 2010, pp. 725-732.
12) S. Rendle, C. Freudenthaler, Z.Gantner, and L. Schmidt-Thieme, "BPR:Bayesian personalized ranking from implicit feedback," in Proc. $25^{\text {th }}$ Conf. Uncertainty Artif. Intell. (UAI), 2009, pp. 452-461.

13) S.-H. Yang, B. Long, A. Smola, N. Sadagopan, Z. Zheng, and H. Zha, "Like like alike: Joint friendship and interest propagation in social networks," in Proc. $W W W, 2011$, pp. 537-546.

14) H. Ma, H. Yang, M. R. Lyu, and I. King, "SoRec: Social recommendation using probabilistic matrix factorization," in Proc. ACM CIKM, 2008, pp. 931-940.

15) M. Jamali and M. Ester, "A matrix factorization technique with trust propagation for recommendation in social networks," in Proc. ACM RecSys, 2010, pp. 135-142

16) X. Yang, H. Steck, and Y. Liu, "Circle-based recommendation in online social networks," in Proc. ACM KDD, 2012, pp. 1267-1275.

17) M. Jiang, P. Cui, X. Chen, F. Wang, W. Zhu, and S. Yang, "Social recommendation with crossdomain transferable knowledge," IEEE Trans.Knowl. Data Eng., vol. 27, no. 11, pp. 3084-3097, Nov. 2015. 